INNOVATIONS IN PRIMARY CARE

\title{
Electronic Medical Record-Based Referrals to Community Nutritional Assistance for Food-Insecure Patients
}

\author{
Kurt Hager, MS \\ Alexandra De Kesel Loftbus, MANM \\ Betty Balan \\ Diana Cutts, MD
}

Ann Fam Med 2020;18:278. https://doi.org/10.1370/afm.2530.

\section{THE INNOVATION}

Food insecurity, defined as the lack of consistent access to enough food for an active, healthy life, has consistently been associated with poor health outcomes and higher health care costs. ${ }^{1}$ To address this issue, a food resource referral was integrated into Hennepin County Medical Center's (HCMC) electronic medical record (EMR) at the hospital, outpatient clinics, and community primary care clinics. The referral order is auto-faxed to staff at a partner food bank, Second Harvest Heartland, and referred patients receive individualized, overthe-phone enrollment in nutrition programs and community food resources. This clinic-to-community linkage fits into clinicians' workflow with minimal disruption, efficiently connecting patients to the hunger relief system.

\section{WHO AND WHERE}

Hennepin County Medical Center is an urban, safety-net medical center and Second Harvest Heartland is a member of Feeding America. Both organizations are in Minneapolis, Minnesota.

\section{HOW}

In 2015, HCMC clinicians began using the Hunger Vital Sign, validated for clinical settings, to identify patients with food insecurity. ${ }^{2}$ Patients who screen positive are offered an electronic referral to the food bank. To remain compliant with the Health Insurance Portability and Accountability Act of 1996 (HIPAA), stored patient information is not transmitted. Clinicians document consent and manually type the patient's pre-

Conflicts of interest: autbors report none.

\section{CORRESPONDING AUTHOR}

Kurt Hager, MS

Tufts University Friedman School of Nutrition Science and Policy

150 Harrison Ave

Boston, MA 02111

kurt.hager@tufts.edu ferred contact information and language into the referral. The order is auto-faxed to the food bank where staff call patients within 24 to 48 hours, attempting a maximum of 3 contacts within 10 days. Food bank staff provide individualized, overthe-phone application assistance for federal nutrition programs, plus information about community resources including food pantries, meal programs, and produce distributions.

We initially were unable to standardize food insecurity screening across the medical center. Instead, we trained over 30 clinic-based and hospital-based teams on the food resource referral. Referrals spiked after these trainings; however, they typically decreased after 3 months. To maintain a steady referral volume, we began quarterly reports communicating referral numbers, patient testimonials, and follow-up results. This process was effective but required a considerable amount of labor.

In 2016, we standardized food insecurity screening at 2 outpatient clinics, Senior Care and Pediatrics. These clinics used the same EMR food resource referral; however, screening occurred at intake in Senior Care and during the annual wellchild visits in Pediatrics. After we introduced systematic screening, Senior Care referrals increased by $1,450 \%(P<.001)$ within 3 months and Pediatrics recorded a $275 \%$ referral increase $(P$ $<.001)$. The success of these pilots demonstrates that standardized screening can greatly improve identification of food insecurity and increase referrals to community partners.

In total, 4,020 patients were referred by medical center staff from January 1, 2015-December 31, 2017. The food bank successfully contacted $63 \%$ and assisted 2,533 households.

\section{LEARNING}

Food insecurity screening combined with a robust referral system is valuable to clinical care. Direct referral relationships with community organizations can be built through utilization of EMR-based communication. An EMR referral is easily integrated into clinician workflow with minimal time added. Education for the whole care team and standardized screenings are critical to successful uptake of this model.

Submitted July 26, 2019; submitted, revised, October 9, 2019; accepted November 4, 2019.

Key words: food insecurity; information management/informatics: electronic health records; health care delivery/HSR: health disparities; nutrition; primary care issues: patient-centered care; social factors in health and health care; health promotion/disease prevention: screening

For supplemental information, including author affiliations and references, see http://www.AnnFamMed.org/content/18/3/278I suppl/DC1. 